\title{
Anti-inflammatory Effects of UDCA on Splenocytes Exposed to PMA/lonomycin
}

\author{
So-Young Parka, Jong Shick Wooc, Yu Jin Jung c, Tae Joon Wonc, Yun Ju HuH', Chan Woo LeE \\ Hyo Shin $\mathrm{KIM}^{\mathrm{c}}$, Seong Soo Joo ${ }^{\mathrm{b}}$, Do Ik LeE ${ }^{\mathrm{c}}$, and Kwang Woo HwANG ${ }^{\mathrm{c} *}$ \\ ${ }^{a}$ Environmental Toxico-Genomic \& Proteomic Center, College of Medicine, Korea University, \\ Anamdong 5, Sungbuk ku, Seoul 136-705, Republic of Korea \\ ${ }^{b}$ Research Institute of Veterinary Medicine, Chungbuk University, Cheongju 361-763, Republic of Korea \\ 'Department of Immunology, College of Pharmacy, Chung-Ang University, \\ 221 Heuksuk-Dong, Dongjak-Ku, Seoul 156-756, Republic of Korea
}

(Received May 28, 2008; Accepted June 14, 2008)

\begin{abstract}
Immunosuppressive therapy after organ transplantation is routinely used to prevent rejection of the organ, because this decreases the risk of adverse events, infection, and malignancies. Recently, ursodeoxycholic acid (UDCA), which is isolated from the dried bile of adult Chinese bears, has been shown to reduce the incidence and severity of acute rejection of liver allograft during early phase of liver transplantation. Therefore, in this study, we investigated the effect of UDCA on the proliferation of splenocytes exposed to PMA plus ionomycin. Our results demonstrated that UDCA decreased the splenocytes' proliferation in a dose-dependent manner. The decreased cell proliferation was accompanied with the decreased secretion of cytokines such as IL-2, IFN- $\gamma$ and TNF- $\alpha$. In addition, the pretreatment of UDCA on splenocytes stimulated with PMA plus ionomycin decreased the mRNA levels of cytokines (IL-2, IFN- $\gamma$ and TNF- $\alpha$ ) and costimulatory molecules (B7.2 and PD-L1). These results suggest the beneficial effect of UDCA on organ transplantation by decreasing lymphocyte proliferation.
\end{abstract}

Keywords: ursodeoxycholic acid, Immunosuppressive therapy, splenocytes, cytokines, costimulatory molecules

\section{INTRODUCTION}

Immunosuppressive therapy after organ transplantation is routinely used to prevent rejection of the organ, because this decreases the risk of adverse events, infection, and malignancies (Rubin, 1987). In case of human liver allografts, its susceptibility to rejection is lower than other organs (Wall and Ghent, 1995), but increasing number of reports regarding to the rejection indicate that immunosuppressive regimens are necessary and beneficial for liver transplantation (Girlanda et al., 2005). Early immunosuppressive regimens used in liver transplantation consisted of cyclosporine A (CsA), a calcinurin inhibitor. Even though CsA has remained the basis of early immunosuppressive protocols, a notable incidence of acute rejection episodes requires the modifications of regi-

\footnotetext{
${ }^{*}$ Corresponding author

Tel: +82-2-820-5597, Fax: +82-2-823-5597

E-mail: khwang@cau.ac.kr
}

mens (Casanovas Taltavull, 2004). The clinical symptoms such as nephrotoxicity, and infectious, malignant complications limit the long-term implication of this drug.

Ursodeoxycholic acid (UDCA, Fig. 1) is a bile acid that is present in human bile at low concentration, and is isolated from the dried bile of adult Chinese bears (Ikegami and Matsuzaki, 2008). Since mid 1980s, UDCA has been used widely in a variety of biliary and liver diseases (Poupon et al., 1997; Tomida et al., 1999). Particularly, UDCA is reported to induce biochemical, histological, and prognostic improvement in patients with primary biliary cirrhosis without adverse reactions (Heathcote, 2000). UDCA has also been shown to reduce the incidence and severity of acute rejection of liver allograft during early phase of liver transplantation (Persson et al., 1990). Despite the supportive effect of UDCA, the target molecules and the mechanisms of action remain unknown.

In this study, we investigated the effect of UDCA on the proliferation of splenocytes stimulated by PMA plus ionomycin. Our results demonstrated that UDCA decreased 
the splenocytes' proliferation in a dose-dependent manner. The decreased cell proliferation was accompanied with the decreased secretion of cytokines such as IL-2, IFN- $\gamma$ and TNF- $\alpha$. In addition, the pretreatment of UDCA on splenocytes stimulated with PMA plus ionomycin decreased the mRNA levels of cytokines (IL-2, IFN- $\gamma$ and TNF- $\alpha$ ) and costimulatory molecules (B7.2 and PD-L1).

\section{MATERIALS AND METHODS}

\section{Chemicals and reagents}

Ursodeoxycholic acid (UDCA), cyclosporine A (CsA), MTT (3-[4,5-dimethylthiazol-2-yl]-2,5-diphenyl tetrazolium bromide), isopropyl alcohol, and DMSO (N,N-dimethyl sulfoxide) were purchased from Sigma (St Louis, MO, USA). Dulbecco's modified Eagle's medium (DMEM), fetal bovine serum (FBS), and penicillin and gentamycin were also obtained from Sigma.

\section{Animals}

C57BL/6 mice were obtained from Harlan \& Winkelmann and kept under conditions of group housing (5 animals per cage), with food and water supplied ad libitum and $12 \mathrm{~h}$ light/dark illumination. All experimental components described were performed in accordance with guidelines established by the Chung-Ang University College of Pharmacy Animal Welfare and Safety program.

\section{Experimental animals and splenocyte culture}

Female 7-8 weeks C57BL/6 mice were sacrificed and spleens isolated. Single cell suspension of splenocytes was obtained after passing tissues of spleen through $100 \mu \mathrm{m}$ nylon mesh. Then, splenocytes were cultured in Dulbecco's modified Eagle's medium (DMEM) supplemented with $10 \%$ fetal bovine serum (FBS).

\section{Determination of UDCA's ability to protect spleno- cytes proliferation}

The ability of UDCA to protect mouse splenocytes from PMA/lonomycin were determined using MTT assays which measure the potential of the cells to reduce MTT to MTT formazan, a reflection of viability. Briefly, cultured splenocytes were plated in 24-well tissue culture plates overnight $\left(5 \times 10^{5} \mathrm{cell} / \mathrm{s} / \mathrm{ml}\right)$. Cells pretreated with different concentrations of UDCA or cyclosporine $(1 \mu \mathrm{g} / \mathrm{ml})$ as a control for $12 \mathrm{~h}$ incubation, were incubated with PMA/ lonomycin ( $50 \mathrm{ng} / \mathrm{ml}$ and $500 \mathrm{ng} / \mathrm{ml}$, respectively). After a $24 \mathrm{~h}$ incubation, MTT solution $(10 \mu \mathrm{L}$ per well of a $5 \mathrm{mg} /$ $\mathrm{ml}$ stock solution in PBS) was added for $4 \mathrm{~h}$ at $37^{\circ} \mathrm{C}$, and cells were subsequently lysed in the presence of $100 \mu \mathrm{l}$ lysis buffer (isopropyl alcohol-0.01N HCl). Optical density of the resulting solutions was colorimetrically determined at $570 \mathrm{~nm}$ using a microplate reader (Molecular Devices; Ramsey, MN, USA).

\section{Proinflammatory and cytokine ELISA}

Cytokine production of splenocytes was assessed using enzyme-linked immunosorbent assay using the method described by (Hwang et al., 2004). Briefly, cultured splenocytes were plated in 24-well tissue culture plates $\left(5 \times 10^{5}\right.$ cells $\left./ \mathrm{ml}\right)$ overnight. Cells pretreated with different concentrations of UDCA or cyclosporine $(1 \mu \mathrm{g} /$ $\mathrm{ml}$ ) for 30 min., were incubated with PMA/lonomycin (50 $\mathrm{ng} / \mathrm{ml}$ and $500 \mathrm{ng} / \mathrm{ml}$, respectively). Supernatants were harvested $24 \mathrm{~h}$ after exposure of splenocytes to PMA/lonomycin. Alternatively, 96-well plates were coated overnight at $4^{\circ} \mathrm{C}$ with anti-IL-2 $\left(1 \mu \mathrm{g} / \mathrm{ml}\right.$ in $0.1 \mathrm{M} \mathrm{NaHCO}_{3} ; \mathrm{pH}$ 8.2) or anti-INF- $\gamma\left(2 \mu \mathrm{g} / \mathrm{ml}\right.$ in $0.1 \mathrm{M} \mathrm{NaHCO}{ }_{3} ; \mathrm{pH}$ 8.2), and washed twice with PBS and $0.05 \%$ Tween 20 before the addition of harvested supernatants. Concentration of cytokines in each sample was detected by ELISA using $\mathrm{Ab}$ pairs as instructed by the manufacturer (BD Pharmingen). Absorbance was detected at $405 \mathrm{~nm}$ in a 96-well spectrophotometer (Molecular Probes), and data were analyzed by comparison to a standard curve generated using recombinant cytokines at known concentrations.

\section{Measurement of mRNA levels by RT-PCR}

Total RNA was prepared from splenocytes using TRIzol reagent (Invitrogen; Carlsbad, CA, USA) following the manufacturer's instructions. Aliquots of total RNA $(1 \mu \mathrm{g})$ were reverse transcribed using oligo (dT) and Superscript II-Reverse Transcriptase (Invitrogen). cDNA templates for PCR amplication using a thermocycler (Applied Biosystems, Foster city, CA, USA) were incubated with the following primers (IL-2, sense; CCCACTTCAAGCTCCACTTC, anti-sense; TCTCAGCTG TGG TGGTGAAG, IFN- $\alpha$, sense; TTTGAGGTCAACAACCCACA, anti-sense; CGCAAT CACAGTCTTGGCTA, TNF- $\alpha$, sense; GTGCCAGCCGATGGGTTGTACC, anti-sense; AGGCCCACA GTCCAGGTCACTG, PD-L1, sense; TGC TGCATAATCAGC TACGG, anti-sense; GAGGAG GACCGTGGACACTA, B7.2, sense; TCCAACAGC CTCTCTCTT TC, anti-sense; TCACTCTGCATTTGGTTTTG. The housekeeping gene, $\alpha$-actin were used for the internal control for each experiment.

\section{Data analysis}

All data in the text and figures are expressed as the mean \pm SD. Statistical analyses were performed using 
Student's $t$ test. Differences were considered statistically significant when $p$ was $<0.05$.

\section{RESULTS}

\section{UDCA induced the cytotoxicity on splenocytes}

Splenocytes are the mixture of white blood cells, and are characteristically purified from the splenic tissues. In this study, we examined the effect of UDCA on the cell proliferation of splenocytes induced by PMA/lonomycin.

We first examined the cytotoxic effects of UDCA on splenocytes. Splenocytes plated on 24-well plates were treated with different concentrations of UDCA. After $24 \mathrm{~h}$ incubation, the cytotoxicity of UDCA on splenocytes was determined using an MTT assay. Treatment of 100-250 $\mu \mathrm{g} / \mathrm{ml}$ UDCA significantly decreased the cell viability of splenocytes, while that of $50 \mu \mathrm{g} / \mathrm{ml}$ UDCA didn't induce the cytotoxicity on splenocytes (Fig. 1).

\section{UDCA decreased the cell proliferation of murine splenocytes stimulated by PMA/lonomycin in a dose-dependent manner}

Next, we evaluated the effect of UDCA on cell proliferation of splenocytes using an MTT assay. Splenocytes were pretreated with different concentrations of UDCA or CsA $(1 \mu \mathrm{g} / \mathrm{ml})$ for $30 \mathrm{~min}$. We used CsA (cyclosporine A) in order to compare the magnitude of UDCA effect on cell proliferation and cytokine suppression. Then, cells were stimulated with PMA (50 ng/ml) plus ionomycin (500 ng/

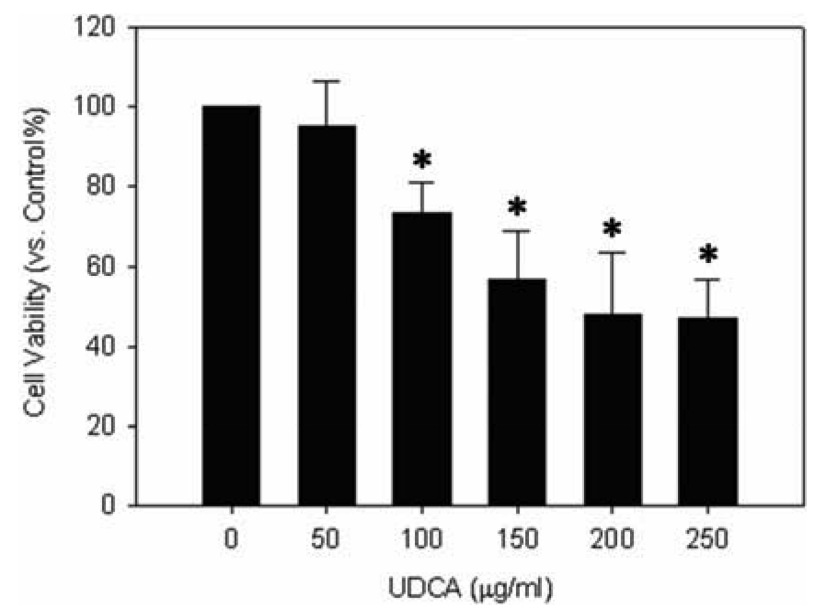

Fig. 1. Effect of UDCA on cell viability in murine splenocytes. Splenocytes treated with different concentrations of UDCA were applied for an MTT assay to estimate the cell viability. All data shown represents as the means $\pm S D$. The values were obtained from three independent experiments. ${ }^{*} p<0.05$, different from control groups.

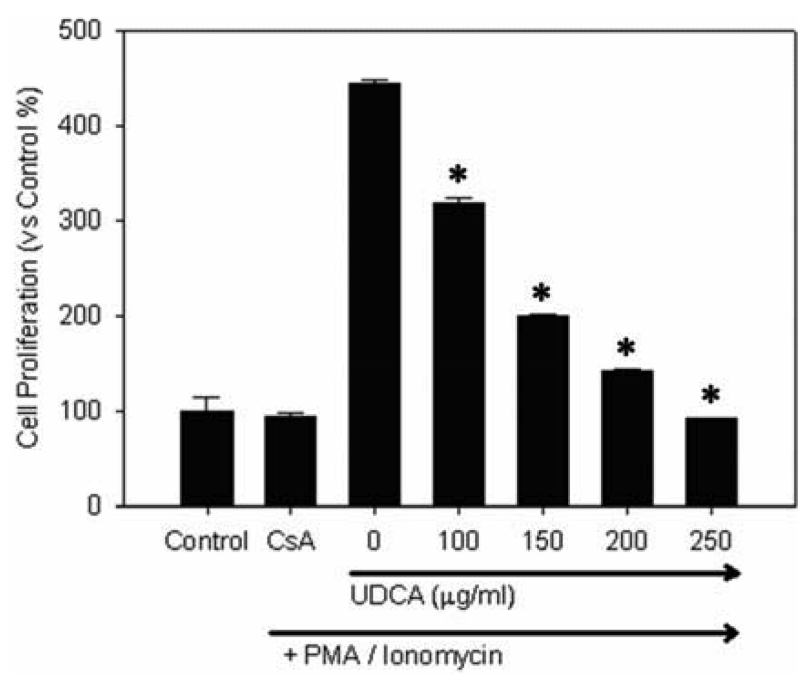

Fig. 2. The effect of UDCA on the proliferation of splenocytes stimulated with PMA/lonomycin. Splenocytes pretreated with different concentrations of UDCA for $12 \mathrm{~h}$ were incubated with PMA (50 ng/ml) plus ionomycin $(500 \mathrm{ng} / \mathrm{ml})$ for an additional $24 \mathrm{~h}$ and the cell viabilities were then estimated by an MTT assay. Note that pretreatment with UDCA dose-dependently decreased the cell proliferantions stimulated by the treatment of PMA plus ionomycin. Cyclosporin $(1 \mu \mathrm{g} / \mathrm{ml})$ was used as a positive control. All data shown represent as the means \pm SD. The values were obtained from three independent experiments. ${ }^{*} p<0.05$, different from the PMA/ionomycin alone treated groups.

$\mathrm{ml}$ ) for $24 \mathrm{~h}$ (Ritter et al., 2005), and the cell proliferation was determined using an MTT assay (Fig. 2). As expected, CsA completely blocked the cell proliferation induced by PMA plus ionomycin. The pretreatment of UDCA also significantly decreased the cell proliferation in a dosedependent manner. Particularly, treatment of $250 \mu \mathrm{g} / \mathrm{ml}$ UDCA almost completely inhibited the cell proliferation induced by PMA plus ionomycin.

\section{UDCA suppressed cytokine synthesis on spleno- cytes stimulated by PMA and ionomycin in a dose- dependent manner}

Next, we investigated the effect of UDCA on secrestion of IL-2, which is important in the proliferation and activation of T cells, using murine splenocytes. Splenocytes were stimulated with PMA plus ionomycin for $24 \mathrm{~h}$ after pretreatment with different concentrations of UDCA or CsA. Then, supernatants were harvested and the secretion of cytokines (IL-2 and IFN- $\gamma$ ) was determined using ELISA method. As shown in Fig. 3, the concentrations of IL-2 and IFN- $\gamma$ in supernatants of control splenocytes and CsA treated splenocytes were not detectable. On the other hand, the secretions of IL-2 and IFN- $\gamma$ were 
A

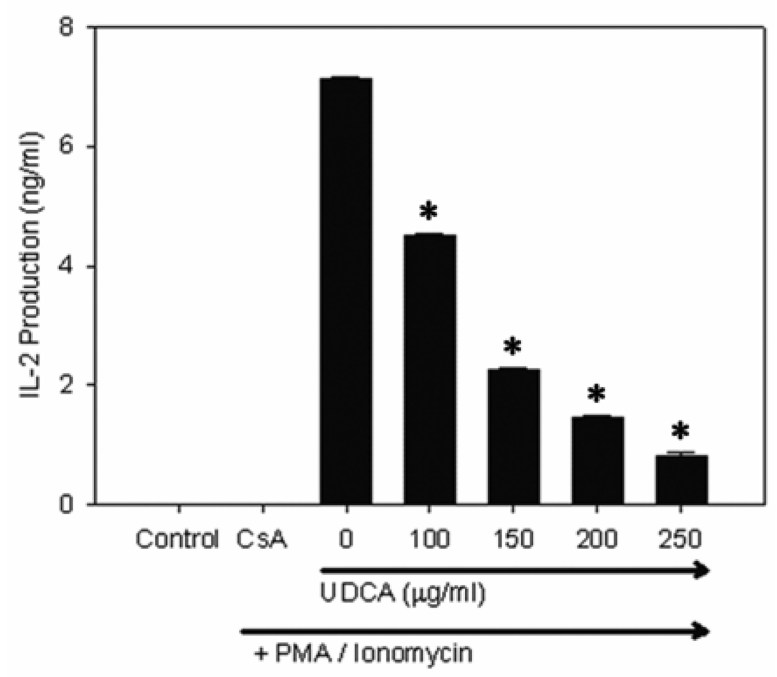

B

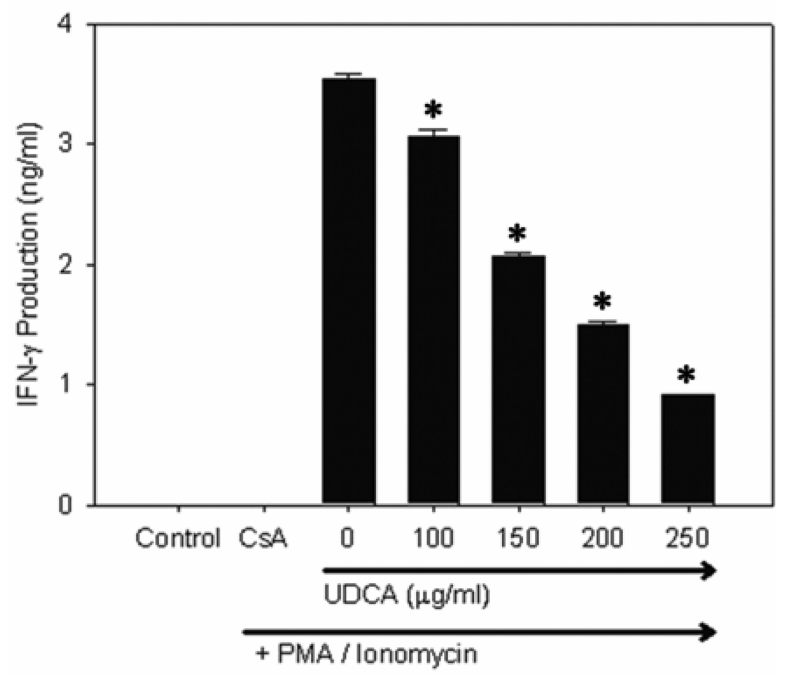

Fig. 3. Effect of UDCA on IL-2 secretion in PMA/lonolycin stimulated splenocytes. Splenocytes pretreated with different concentrations of UDCA were stimulated by PMA/lonomycin. The secretion of IL-2 (A) and IFN- $\gamma(B)$ were measured using an ELISA. Cyclosporin $(1 \mu \mathrm{g} / \mathrm{ml})$ was used as a positive control. All data shown represent as the means \pm SD. The values were obtained from three independent experiments.

${ }^{*} p<0.05$, different from the PMA/ionomycin alone treated groups.

dramatically increased in supernatants of splenocytes treated with PMA/lonomycin alone, where as the increases of cytokine concentrations stimulated by PMA/lonomycin were significantly decreased by the pretreatment with UDCA in a dose-dependent manner. These results suggest that UDCA decreased T cell activation and proliferation by the inhibition of cytokine production.

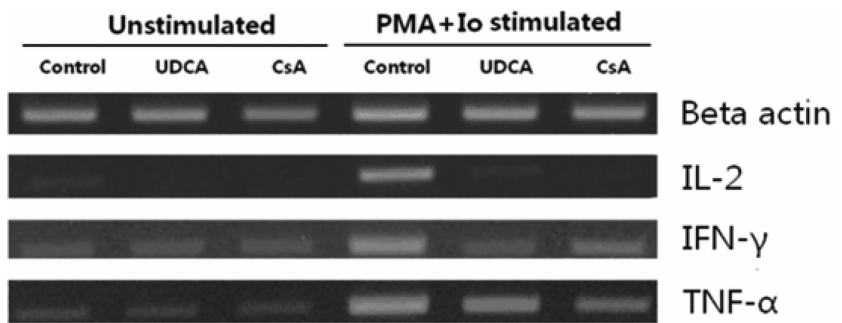

Fig. 4. The effect of UDCA on cytokine mRNA levels in PMA/ lonomycin stimulated splenocytes. Splenocytes pretreated with UDCA $(250 \mu \mathrm{g} / \mathrm{ml})$ were stimulated by PMA and ionomycin for $24 \mathrm{~h}$. The changes of mRNA levels of IL-2, IFN- $\gamma$, and TNF- $\alpha$ were evaluated by RT-PCR. Cyclosporin $(1 \mu \mathrm{g} / \mathrm{ml})$ was used as a positive control.

\section{UDCA decreased pro-inflammatory cytokine mRNA levels}

Next, we investigated whether pretreatment with UDCA decreased the cytokine mRNA levels in splenocytes stimulated by PMA/lonomycin. As shown in Fig. 4, cytokine mRNA levels in unstimulated control splenocytes were very low, and treatment of unstimulated splenocytes with UDCA or CsA didn't increase the expression of cytokine mRNA levels. In contrast, the stimulation of control splenocytes with PMA/lonomycin dramatically increased the mRNA levels of IL-2, IFN- $\gamma$, and TNF- $\alpha$. Conversely, pretreatment of splenocytes with CsA $(1 \mu \mathrm{g} / \mathrm{ml})$ before the stimulation with PMA/lonomycin significantly decreased the expression of cytokine mRNAs as compared to PMA/ lonomycin stimulated control splenocytes. Same as CsA treated cells, cytokine mRNA levels decreased significantly in UDCA $(250 \mu \mathrm{g} / \mathrm{ml})$ pretreated splenocytes in advance of PMA/lonomycin stimulation. UDCA is more potent in suppressing IL-2, while it is less effective in suppressing in TNF- $\alpha$.

\section{UDCA decreased co-stimulatory molecule mRNA levels}

It has been known that costimulatory molecule, B7.2 is responsible for the activation of $\mathrm{T}$ cells by some stimuli (Wahl et al., 2002), while PD-L1 plays an inhibitory role in regulating $T$ cell activation in the periphery, and is highly expressed on inflammatory macrophages (Loke and Allison, 2003). In order to evaluate the effects of UDCA on the expression of costimulatory molecules in splenocytes stimulated by PMA/lonomycin, the mRNA levels of B7.2 and PD-L1 were determined using RT-PCR methods (Fig. 5). B7.2 and PD-L1 mRNA levels were very low in unstimulated control and UDCA alone treated splenocytes, while the treatment of splenocytes with CsA with- 


\begin{tabular}{|lllll}
\multicolumn{2}{c}{ Unstimulated } & & PMA + Io stimulated & \\
\hline Control UDCA CSA & Control UDCA CSA & Beta actin \\
\hline & & & PDL1 \\
\hline & & & B7.2
\end{tabular}

Fig. 5. The effect of UDCA on mRNA levels of costimulatory molecules, B7.2, and PD-L1, in PMA/lonomycin stimulated splenocytes. Splenocytes pretreated with UDCA $(250 \mu \mathrm{g} / \mathrm{ml})$ were stimulated by PMA and ionomycin for additional $24 \mathrm{~h}$. The changes of mRNA levels of B7.2 and PD-L1 were evaluated by RT-PCR. Cyclosporin $(1 \mu \mathrm{g} / \mathrm{ml})$ was used as a positive control.

out stimulation increased mRNA levels of both B7.2 and PD-L1. On the other hand, stimulation of splenocytes with PMA/lonomycin increased both mRNA levels compared to unstimulated splenocytes. However, pretreatment of splenocytes with UDCA before the stimulation significantly decreased the B7.2 and PD-L1 mRNA levels compared to stimulated splenocytes, where as CsA treatment prior to the stimulation rather increased both mRNA levels compared to stimulated splenocytes. These results indicate that the mechanisms of $T$ cell inactivation induced by UDCA are different from those by CsA.

\section{DISCUSSION}

UDCA which is isolated from the dried bile of adult Chinese bears, has been used widely in a variety of biliary and liver diseases since mid 1980s. Particularly, UDCA has reduced the incidence and severity of acute rejection of liver allograft during early phase of liver transplantation (Persson et al., 1990). However, the target molecules and the mechanisms of action remain unknown.

In this study, we demonstrated UDCA decreased the splenocytes' proliferation by the inhibition of cytokine and co-stimulatory molecule production induced by PMA plus ionomycin. UDCA has been reported to suppress the secretion of IL-2, IL-4 and INF- $\gamma$ from activated T lymphocytes (Tanaka et al., 1996). The immunosuppression by orally administered UDCA enhanced liver regeneration in partially hepatectomized rats (Liu et al., 2002).

IL-2 is a cytokine released by activated T-cells, while IFN- $\gamma$ is mainly produced by T-cells and NK-cells. On the other hand, TNF- $\alpha$ is mainly derived from monocytes and macrophages (Borish and Steinke, 2003). In our study, UDCA suppressed the expression of IL-2 and INF- $\gamma$ more efficiently than that of TNF- $\alpha$ in splenocytes. Since splenocytes are the mixture of white blood cells, these results suggest that UDCA works on $\mathrm{T}$ cells more effectively than macrophages.

Activation of $T$ cells occurs through the engagement of both the $T$ cell receptor and CD28 on the $T$ cell by the major histocompatibility complex peptide and B7 family members on the APC, respectively. The signaling pathways induce the released calcium, followed by the activation of calcineurin. This eventually activates the transcription of $\mathrm{L}-2$, a cytokine that promotes long term proliferation of activated T cells. In this study, we employed PMA (phorbol 12-myristate 13-acetate) and ionomycin (Calcium ionophore) as stimulants. PMA and ionomycin has been well known to increase the expression of IL-2 and IFN- $\gamma$ in T cells, which are two necessary lymphokines in the development of cytolytic T cells (Kelso and Gough, 1989; Maraskovsky et al., 1989). Our results indicated that the treatment of PMA plus ionomycin on splenocytes increased the expressions of IL-2, IFN- $\gamma$ and TNF- $\alpha$, which is in agreement with previous reports (Kelso and Gough, 1989; Maraskovsky et al., 1989). Furthermore, the pretreatment of UDCA in advance of PMA plus ionomycin suppressed the production of those cytokines compared to PMA and ionomycin alone treated cells. On the other hand, UDCA pretreatment on splenocytes prior to PMA and ionomycin decreased the mRNA levels of B7.2 and PD-L1 compared to PMA and ionomycin alone treated cells. B7.2 is one of costimulatory molecules which is involved in activating T cells (Brown et al., 2003), while PD-L1 is to inhibit T cell proliferation (Loke and Allison, 2003). These results suggest that the suppression of splenocytes' proliferation induced by UDCA is through the inhibition of cytokine production and co-stimulatory molecue, B7.2, but not through PD-L1.

In summary, the pretreatment of UDCA on splenocytes inhibited the cell proliferation stimulated by PMA and ionomycin. This effect was mediated by the inhibition of cytokine production, and B7.2, costimulatory molecule. These results support the beneficial effects of UDCA on organ transplantation by reducing the lymphocytes' proliferation, and eventually organ rejection.

\section{ACKNOWLEDGEMENTS}

This work was supported by Korea Research Foundation Grant funded by Korea Government (MOEHRD, Basic Research Promotion Fund) (KRF-2005-003-E00077).

\section{REFERENCES}

Borish, L.C. and Steinke, J.W. (2003). 2. Cytokines and chemokines. J Allergy Clin Immunol 111, S460-475. 
Brown, J.A., Dorfman, D.M., Ma, F.R., Sullivan, E.L., Munoz, O., Wood, C.R., Greenfield, E.A. and Freeman, G.J. (2003). Blockade of programmed death-1 ligands on dendritic cells enhances $\mathrm{T}$ cell activation and cytokine production. J Immunol 170, 1257-1266.

Casanovas Taltavull, T. (2004). Impact of cyclosporine on the development of immunosuppressive therapy in liver transplantation. Transplant Proc 36, 291S-294S.

Girlanda, R., Rela, M., Williams, R., O'Grady, J.G. and Heaton, N.D. (2005). Long-term outcome of immunosuppression withdrawal after liver transplantation. Transplant Proc 37, 17081709.

Heathcote, E.J. (2000). Management of primary biliary cirrhosis. The American Association for the Study of Liver Diseases practice guidelines. Hepatology 31, 1005-1013.

Hwang, K.W., Sweatt, W.B., Mashayekhi, M., Palucki, D.A., Sattar, H., Chuang, E. and Alegre, M.L. (2004). Transgenic expression of CTLA-4 controls lymphoproliferation in IL-2deficient mice. J Immunol 173, 5415-5424.

Ikegami, T. and Matsuzaki, Y. (2008). Ursodeoxycholic acid: Mechanism of action and novel clinical applications. Hepatol Res 38, 123-131.

Kelso, A. and Gough, N.M. (1989). Differential inhibition by cyclosporin a reveals two pathways for activation of lymphokine synthesis in T cells. Growth Factors 1, 165-177.

Liu, L., Sakaguchi, T., Cui, X., Shirai, Y., Nishimaki, T. and Hatakeyama, K. (2002). Liver regeneration enhanced by orally administered ursodesoxycholic acid is mediated by immunosuppression in partially hepatectomized rats. $A m \mathrm{~J}$ Chin Med 30, 119-126.

Loke, P. and Allison, J.P. (2003). PD-L1 and PD-L2 are differentially regulated by Th1 and Th2 cells. Proc Natl Acad Sci USA 100, 5336-5341.
Maraskovsky, E., Chen, W.F. and Shortman, K. (1989). IL-2 and IFN-gamma are two necessary lymphokines in the development of cytolytic T cells. J Immunol 143, 1210-1214.

Persson, H., Friman, S., Schersten, T., Svanvik, J. and Karlberg, I. (1990). Ursodeoxycholic acid for prevention of acute rejection in liver transplant recipients. Lancet 336, 52-53.

Poupon, R.E., Lindor, K.D., Cauch-Dudek, K., Dickson, E.R., Poupon, R. and Heathcote, E.J. (1997). Combined analysis of randomized controlled trials of ursodeoxycholic acid in primary biliary cirrhosis. Gastroenterology 113, 884-890.

Ritter, M., Hohenberger, K., Alter, P., Herzum, M., Tebbe, J. and Maisch, M. (2005). Caffeine inhibits cytokine expression in lymphocytes. Cytokine 30, 177-181.

Rubin, R.H. (1987). The compromised host as sentinel chicken. N Engl J Med 317, 1151-1153.

Tanaka, H., Makino, Y., Miura, T., Hirano, F., Okamoto, K., Komura, K., Sato, Y. and Makino, I. (1996). Ligand-independent activation of the glucocorticoid receptor by ursodeoxycholic acid. Repression of IFN-gamma-induced MHC class II gene expression via a glucocorticoid receptor-dependent pathway. J Immunol 156, 1601-1608.

Tomida, S., Abei, M., Yamaguchi, T., Matsuzaki, Y., Shoda, J., Tanaka, N. and Osuga, T. (1999). Long-term ursodeoxycholic acid therapy is associated with reduced risk of biliary pain and acute cholecystitis in patients with gallbladder stones: a cohort analysis. Hepatology 30, 6-13.

Wahl, P., Schoop, R., Bilic, G., Neuweiler, J., Le Hir, M., Yoshinaga, S.K. and Wuthrich, R.P. (2002). Renal tubular epithelial expression of the costimulatory molecule B7RP-1 (inducible costimulator ligand). J Am Soc Nephrol 13, 1517-1526.

Wall, W.J. and Ghent, C.N. (1995). Immunosuppression in liver transplantation: monitoring, dose adjustment, reduction, and withdrawal. Liver Transpl Surg 1, 3-10. 